\title{
Are intraoperative abdominal radiographs necessary for identification of clips during totally laparoscopic or robotic gastrectomy?
}

Jeong Eun Seo*, Sung Eun Oh, Jeong Eun Seo*, Ji Yeong An, Min-Gew Choi, Tae Sung Sohn, Jae Moon Bae, Sung Kim, Jun Ho Lee Department of Surgery, Samsung Medical Center, Sungkyunkwan University School of Medicine, Seoul, Korea

Purpose: Preoperative endoscopic clipping is a popular method in identifying the location of tumors during total laparoscopic or robotic gastrectomy. We investigated the usefulness of additional intraoperative abdominal radiographs to identify the location of clips.

Methods: We retrospectively analyzed 331 patients with early gastric cancer who underwent endoscopic clipping before total laparoscopic or robotic gastrectomy between September 2012 and September 2018. Endoscopists applied two clips $1 \mathrm{~cm}$ from the proximal aspect of the upper margin of the tumor which was located above the angle of the stomach. We compared outcomes of patients who underwent preoperative abdominal radiographs only (group A) and those who underwent additional abdominal radiographs (group B).

Results: Of the total patients, 80 (24.2\%) underwent additional intraoperative abdominal radiographs. The rates of positive frozen biopsy in the two groups were not significantly different (group A vs. group B: $2.8 \%$ vs. $3.8 \%, P=0.456$ ). The additional resection rate was significantly higher in group $B$ compared to group $A$ ( $8.8 \%$ vs. $2.8 \%, P=0.048)$. The mean distance from the tumor was $3.3 \mathrm{~cm}( \pm 2.4)$ in group $A$ and 2.4 $\mathrm{cm}( \pm 1.3)$ in group $B(P<0.001)$. Large tumor size $(\geq 2.4 \mathrm{~cm})$ was significantly associated with additional resection (odds ratio, $5.53 ; 95 \%$ confidence interval, 1.17-26.30; $P=0.031$.

Conclusion: Additional intraoperative abdominal radiographs may be unnecessary for confirmation of proximal resection margin, if the resection line can be predetermined with preoperative abdominal radiographs. For large tumors, to avoid additional resection, the resection line should be placed $1 \mathrm{~cm}$ or more proximally from the preoperatively applied clips.

Keywords: Early gastric cancer, Laparoscopy, Gastrectomy, Abdominal radiograph, Resection margin

Received: Aug 2, 2019 Revised: Nov 1, 2019 Accepted: Dec 5, 2019

Correspondence to: Jun Ho Lee

Department of Surgery, Samsung Medical Center, Sungkyunkwan

University School of Medicine, 81 Irwon-ro, Gangnam-gu, Seoul 06351,

Korea

Tel: +82-2-3410-3463, Fax: +82-2-3410-6981

E-mail: gsjunholee@gmail.com

ORCID: Jeong Eun Seo (https://orcid.org/0000-0003-2261-2932), Sung Eun Oh (https://orcid.org/0000-0003-1195-0602), Jeong Eun Seo (https://orcid.org/00000003-1261-4467), Ji Yeong An (https://orcid.org/0000-0003-1690-4947), MinGew Choi (https://orcid.org/0000-0003-3394-5177), Tae Sung Sohn (https://orcid. org/0000-0002-9553-3234), Jae Moon Bae (https://orcid.org/0000-0003-39280743), Sung Kim (https://orcid.org/0000-0003-0058-8230), Jun Ho Lee (https:// orcid.org/0000-0002-0131-1527)

*These authors have the same name.

Copyright (C) 2019 Korean Society of Surgical Oncology

This is an Open Access article distributed under the terms of the Creative Commons Attribution Non-Commercial License (http://creativecommons.org/licenses/by-nc/4.0) which permits unrestricted non-commercial use, distribution, and reproduction in any medium, provided the original work is properly cited.

\section{INTRODUCTION}

As long-term oncological safety has been confirmed over time, laparoscopic or robotic gastrectomy has become a standard option in treatment of early gastric cancer (EGC) [1,2]. EGC is defined as a malignancy invading the mucosa and submucosal layer, regardless of lymph node metastasis [3]. Because of the shallow depth of the tumor, it is difficult to determine the exact location of the lesion by simply touching the serosa with surgical instruments during total laparoscopic or robot surgery [4]. Therefore, it is important to use an adequate tumor localization method to ensure adequate proximal resection lines during intracorporeal anastomosis.

For total laparoscopic or robotic surgery, there are several methods for localization of the tumor [5-7], such as endoscopic tattooing, intraoperative endoscopy, or ultrasonography. However, the blurring effect of tattoo ink or need for additional equipment and endoscopists for intraoperative endoscopy have made the preop- 
erative endoscopic clipping method the most popular option [4].

The location of the clips can be identified with abdominal radiographs (abdominal X-ray, AXR) before surgery and can be confirmed with intraoperative AXR when the surgeons cannot determine an adequate resection line during the operation. Intraoperative AXR can be performed easily and safely. However, it can be time consuming during surgery, and there are concerns of additional radiation exposure [8].

These disadvantages lead us to question the usage of additional AXR during the operation. Through this study, we compared the outcomes between patients who did not undergo intraoperative AXR before gastric resection and those who did.

\section{METHODS}

\section{Patients and preoperative localization procedure}

Patients ( $\mathrm{n}=926)$ with clinical EGC who underwent laparoscopic or robotic gastrectomy and intracorporeal anastomosis at Samsung Medical Center from September 2012 to September 2018 were enrolled in this study. Among them, 331 patients with tumor above the stomach angle underwent preoperative clipping localization with esophagogastroduodenoscopy (EGD), and more than 2 metal clips were applied approximately $1 \mathrm{~cm}$ above the lesion before or in the early morning of the day of the operation. Immediately after clipping, AXR including supine and erect positions was performed to confirm the location of the clips (Fig. 1).

\section{Intraoperative localization procedure}

During the operations of 251 patients (group A), the location of the clips was estimated based on multiple images of preoperative
EGD and AXR. Intraoperative AXR was not performed before gastric resection for those patients. Landmarks were angled when the tumors were located on the lesser curvature side and gastric rugae when the tumors were located on the greater curvature side. The distances between those landmarks and the tumors were estimated with the length of the endoscopic tube from the esophagogastric junction to the tumors. After gauging the location of the tumors based on preoperative EGD and AXR, laparoscopic metal clips were additionally applied in 80 patients (group B). The metal clips were applied at the greater curvature and lesser curvature of the stomach surface. After intraoperative AXR, a surgeon confirmed whether the endoscopic clips were located between the laparoscopic metal clips (Fig. 2). A line was drawn on the stomach wall to visualize the proximal resection margin (PRM), which was located between the metal clips and at least $2 \mathrm{~cm}$ from the endoscopic clips.

\section{Confirmation of the clips on the specimens}

After resection of the stomach, the specimen was retrieved from the abdominal cavity. After identification of the clips and the tumor, frozen biopsy was performed on the proximal margins to confirm the presence of cancer cells. The PRM $(\mathrm{cm})$ was defined as the shortest length between the tumor and the proximal resection line. If the resection margin was positive for malignancy, additional resection was performed.

We retrospectively analyzed the clinicopathologic characteristics (age, sex, use of intraoperative AXR, tumor location, tumor size, histologic type, type of gastrectomy, and reconstruction) and outcomes (result of frozen biopsy, re-resection, and PRM) of the preoperative clipped patients on the basis of medical records and
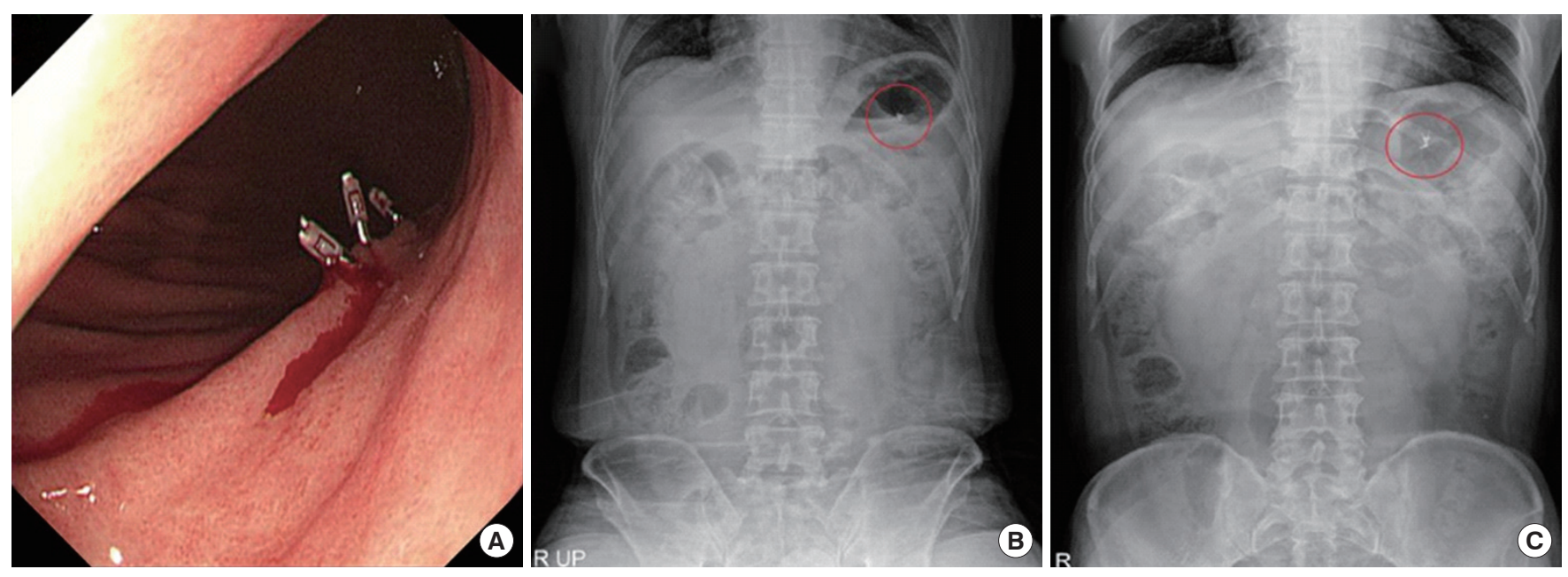

Fig. 1. (A) Preoperatively applied clips during esophagogastroduodenoscopy. (B, C) Abdominal radiographs in erect and supine positions after preoperative endoscopic clipping (red circle). 

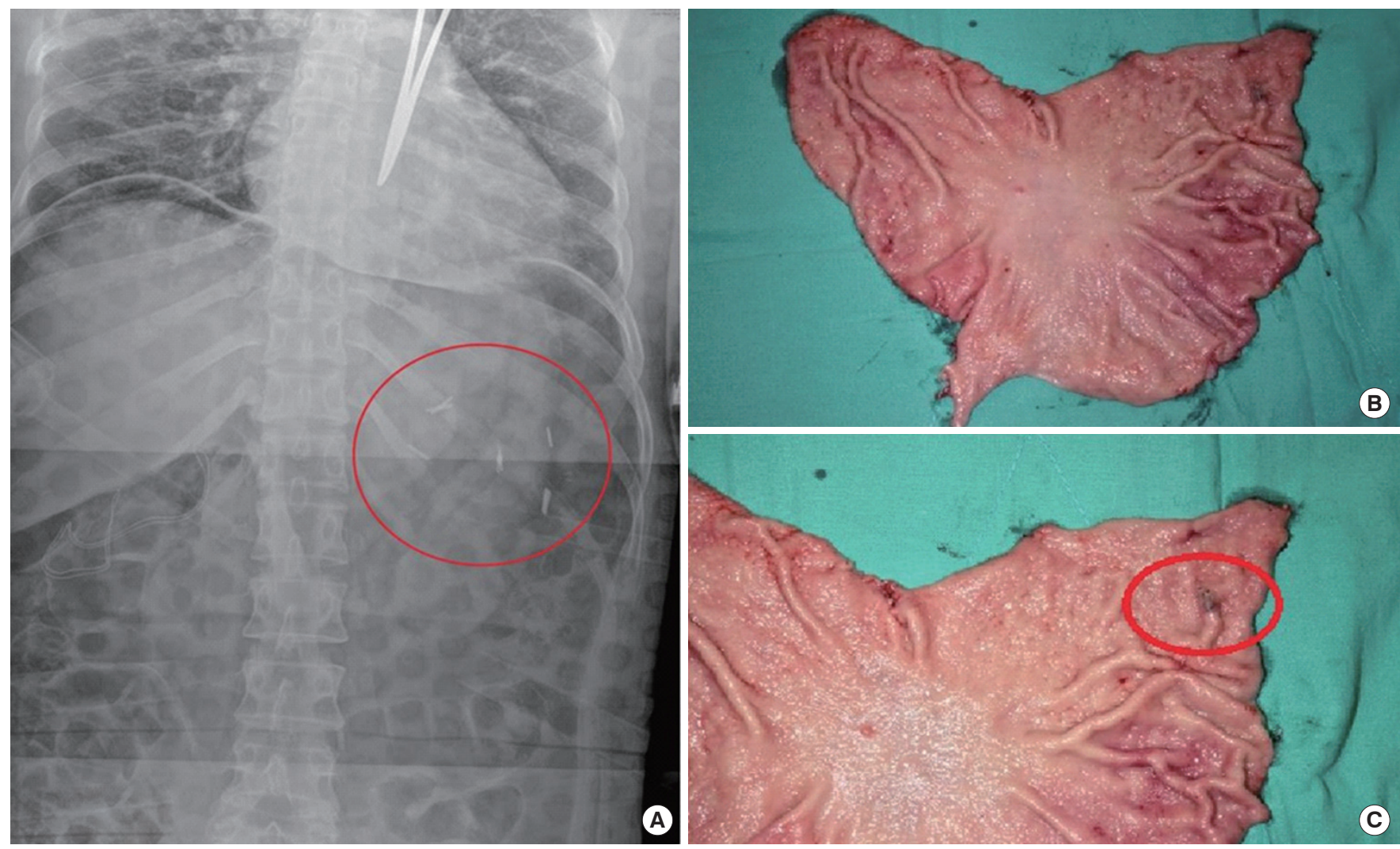

Fig. 2. (A) Intraoperative abdomen radiograph after laparoscopic metal clipping at the lesser and greater curvatures. The preoperative endoscopic applied clips were identified and were located between the metal clips (red circle). (B, C) After gastrectomy, the specimen was removed from the abdominal cavity. After the specimen was cut at the greater curvature aspect, the endoscopic clip (red circle) was found, and the sur-geon confirmed the proximal resection margin with frozen biopsy.

pathologic reviews.

The study was approved by the Institutional Review Board of Samsung Medical Center (IRB No. SMC 2019-01-095).

\section{Statistical analysis}

Differences in clinicopathologic parameters and outcomes between patients in groups A and B were determined by t-test, chisquare test, and Fisher exact test. After evaluating the receiver operating characteristics and area under the curve (ROC and AUC) of the variables significantly associated with outcome, we used Youden indices to determine the optimal cutoff value of the continuous variables. The factors significant in univariate analysis were confirmed with multivariate logistic regression analysis. $\mathrm{P}<0.05$ was considered statistically significant. Statistical analysis was carried out using the statistical software SPSS version 25.0 for Windows (IBM Corp., Armonk, NY, USA).

\section{RESULTS}

The clinicopathologic characteristics, operation data, and out- comes of the preoperative clipped patients $(\mathrm{n}=331)$ are described in Table 1. Tumor location had no significant difference in both groups $\mathrm{A}$ and $\mathrm{B}$, and was distributed mostly at the middle portion of the stomach ( $83.3 \%$ vs. $92.5 \%, \mathrm{P}=0.052)$. Tumor size was also significantly larger in group B compared to group A $(3.2 \mathrm{~cm}$ vs. 2.6 $\mathrm{cm}, \mathrm{P}=0.046)$. Sixty-five percent of the patients in group $B$ underwent Billroth I anastomosis after gastrectomy, and $75.7 \%$ of the patients in group A underwent Billroth II anastomosis $(\mathrm{P}<0.001)$. The rate of positive frozen biopsy in group B was $3.8 \%(n=3)$, while that in group A was $2.8 \%(\mathrm{n}=7)$. The rate of additional resection was significantly higher in group B (7/80, 8.8\%) compared to group $\mathrm{A}(7 / 251,2.8 \%)(\mathrm{P}=0.048)$. The mean length of the PRM was $2.4 \mathrm{~cm}$ in group $B$ and $3.3 \mathrm{~cm}$ in group $A$, a statistically significant difference $(\mathrm{P}<0.001)$.

The factors associated with positive frozen biopsy results and additional resection rate are shown in Table 2. Ten cases (3.0\%) were confirmed with tumor positive frozen biopsy results, and 14 patients (4.2\%) underwent a re-resection procedure during operation. After extraction of the specimen from the abdominal cavity, four patients (1.2\%) had to undergo re-resection due to short PRM 
Table 1. Clinicopathologic characteristics and the outcomes of preoperative clipped EGC patients

\begin{tabular}{|c|c|c|c|c|}
\hline Characteristics & No. of patients $(n=331)$ & Group A $(n=251)$ & Group B $(n=80)$ & P-value ${ }^{\text {a) }}$ \\
\hline \multicolumn{5}{|l|}{ Clinical } \\
\hline Mean age (yr) & & 54.1 & 56.8 & $0.080^{c)}$ \\
\hline Age (yr) & & & & 0.230 \\
\hline$\geq 60$ & 213 & $166(66.1)$ & 47 (58.8) & \\
\hline$<60$ & 118 & 85 (33.9) & 33 (41.2) & \\
\hline Sex & & & & 0.272 \\
\hline Male & 179 & $140(55.8)$ & $39(48.8)$ & \\
\hline Female & 152 & $111(44.2)$ & $41(51.2)$ & \\
\hline \multicolumn{5}{|l|}{ Pathological } \\
\hline Tumor location & & & & $0.052^{b)}$ \\
\hline Lower & 45 & 40 (15.9) & $5(6.2)$ & \\
\hline Middle & 283 & 209 (83.3) & $74(92.5)$ & \\
\hline High & 3 & $2(0.8)$ & $1(1.3)$ & \\
\hline Mean tumor size $(\mathrm{cm})$ & & 2.6 & 3.2 & $0.046^{c)}$ \\
\hline Histologic type & & & & 0.471 \\
\hline Differentiated & 93 & $68(27.1)$ & 25 (31.2) & \\
\hline Undifferentiated & 238 & $183(72.9)$ & 55 (68.8) & \\
\hline Lauren type & & & & 0.481 \\
\hline Intestinal & 85 & 65 (25.9) & $20(25.0)$ & \\
\hline Diffuse & 205 & $158(62.9)$ & 47 (58.8) & \\
\hline Mixed & 41 & $28(11.2)$ & 13 (16.2) & \\
\hline \multicolumn{5}{|l|}{ Surgical } \\
\hline Operator ${ }^{\mathrm{d})}$ & & & & $<0.001$ \\
\hline$J H L$ & 212 & $212(84.5)$ & 0 & \\
\hline JYA & 119 & $39(15.5)$ & $80(100.0)$ & \\
\hline Method of operation & & & & $<0.001$ \\
\hline Laparoscopic & 272 & 232 (92.4) & $40(50.0)$ & \\
\hline Robotic & 59 & $19(7.6)$ & $40(50.0)$ & \\
\hline Gastrectomy & & & & $<0.001^{b)}$ \\
\hline Subtotal & 318 & $248(98.8)$ & 70 (87.5) & \\
\hline Total & 13 & $3(1.2)$ & $10(12.5)$ & \\
\hline Reconstruction & & & & $<0.001^{b)}$ \\
\hline Billroth I & 103 & $51(20.3)$ & $52(65.0)$ & \\
\hline Billroth II & 190 & $190(75.7)$ & 0 & \\
\hline RYEJ & 13 & $3(1.2)$ & $10(12.5)$ & \\
\hline RY GJ & 25 & $7(2.8)$ & $18(22.5)$ & \\
\hline \multicolumn{5}{|l|}{ Outcome } \\
\hline Frozen biopsy & & & & $0.456^{b)}$ \\
\hline Negative & 321 & 244 (97.2) & 77 (96.2) & \\
\hline Positive & 10 & $7(2.8)$ & $3(3.8)$ & \\
\hline Re-resection & & & & $0.048^{b)}$ \\
\hline No & 317 & $244(97.2)$ & 73 (91.2) & \\
\hline Yes & 14 & $7(2.8)$ & 7 (8.8) & \\
\hline Mean PRM (cm) & & 3.3 & 2.4 & $<0.001^{\mathrm{c})}$ \\
\hline
\end{tabular}

Values are presented as number (\%). The patients who underwent intraoperative abdominal radiographs were categorized into group $B_{1}$ and those who did not were sorted into group A.

EGC, early gastric cancer; RY EJ, Roux-en Y esophagojejunostomy; RY GJ, Roux-en Y gastrojejunostomy; PRM, proximal resection margin.

${ }^{a}$ Chi-square test or ${ }^{b)}$ Fisher exact test, except for ${ }^{\mathrm{c}} \mathrm{t}$-test. ${ }^{\mathrm{d}}$ Cases performed by only two surgeons (JHL and JYA) in this study.

$(<0.5 \mathrm{~cm})$, regardless of negative frozen biopsy results.

Two out of four patients underwent additional $2 \mathrm{~cm}$ resection, and frozen biopsy was performed on the PRM to confirm a nega- tive result. The other two patients underwent total gastrectomy because the location of the lesion was proximal midbody, which was close to the cardia. 
Table 2. Factors associated with frozen biopsy results and re-resection procedure

\begin{tabular}{|c|c|c|c|c|c|c|}
\hline \multirow{2}{*}{ Factor } & \multicolumn{3}{|c|}{ Frozen biopsy result } & \multicolumn{3}{|c|}{ Re-resection } \\
\hline & Negative $(n=321)$ & Positive $(n=10)$ & P-value ${ }^{a)}$ & No $(n=317)$ & Yes $(n=14)$ & P-value ${ }^{\text {a) }}$ \\
\hline Tumor location & & & 1.000 & & & 1.000 \\
\hline Lower & 44 (13.7) & $1(10.0)$ & & 43 (13.6) & $2(14.3)$ & \\
\hline Middle & $274(85.4)$ & $9(90.0)$ & & $271(85.5)$ & $12(85.7)$ & \\
\hline High & $3(0.9)$ & 0 & & $3(0.9)$ & 0 & \\
\hline Mean tumor size $(\mathrm{cm})$ & 2.7 & 5.5 & $0.035^{b)}$ & 2.6 & 4.9 & $0.032^{b)}$ \\
\hline Histologic type & & & 0.454 & & & 1.000 \\
\hline Differentiated & 91 (28.6) & $2(20.0)$ & & $89(28.1)$ & $4(28.6)$ & \\
\hline Undifferentiated & $230(71.4)$ & $8(80.0)$ & & $228(71.9)$ & $10(71.4)$ & \\
\hline Lauren type & & & 0.105 & & & 0.517 \\
\hline Intestinal & $84(26.4)$ & $1(10.0)$ & & $82(25.9)$ & $3(21.4)$ & \\
\hline Diffuse & $198(61.5)$ & $7(70.0)$ & & $197(62.1)$ & $8(57.1)$ & \\
\hline Mixed & 39 (12.1) & $2(20.0)$ & & 38 (12.0) & $3(21.4)$ & \\
\hline Method of operation & & & 1.000 & & & 0.083 \\
\hline Laparoscopic & $263(82.0)$ & $9(90.0)$ & & 263 (83.0) & $9(64.3)$ & \\
\hline Robotic & 58 (18.0) & $1(10.0)$ & & $54(17.0)$ & $5(35.7)$ & \\
\hline Gastrectomy & & & 0.005 & & & $<0.001$ \\
\hline Subtotal & 311 (96.6) & $7(70.0)$ & & 309 (97.5) & $9(64.3)$ & \\
\hline Total & $10(3.4)$ & $3(30.0)$ & & $8(2.5)$ & $5(35.7)$ & \\
\hline Reconstruction & & & 0.003 & & & $<0.001$ \\
\hline Billroth I & 103 (32.0) & 0 & & 102 (32.2) & $1(7.1)$ & \\
\hline Billroth II & $183(57.1)$ & $7(70.0)$ & & $183(57.7)$ & $7(50.0)$ & \\
\hline RYEJ & $10(3.1)$ & $3(30.0)$ & & $8(2.5)$ & $5(35.7)$ & \\
\hline RY GJ & $25(7.8)$ & 0 & & $24(7.6)$ & $1(7.1)$ & \\
\hline
\end{tabular}

Values are presented as number (\%).

RY EJ, Roux-en Y esophagojejunostomy; RY GJ, Roux-en Y gastrojejunostomy.

${ }^{a)}$ Fisher exact test, except for ${ }^{b)}$ t-test.

Table 3. Univariate and multivariate analyses of frozen biopsy results and re-resection procedure

\begin{tabular}{|c|c|c|c|c|c|c|c|c|}
\hline \multirow{3}{*}{ Factor } & \multicolumn{4}{|c|}{ Frozen biopsy result } & \multicolumn{4}{|c|}{ Re-resection } \\
\hline & \multicolumn{2}{|c|}{ Univariate } & \multicolumn{2}{|c|}{ Multivariate } & \multicolumn{2}{|c|}{ Univariate } & \multicolumn{2}{|c|}{ Multivariate } \\
\hline & OR $(95 \% \mathrm{Cl})$ & P-value & OR $(95 \% \mathrm{Cl})$ & P-value & OR (95\% Cl) & P-value & OR $(95 \% \mathrm{Cl})$ & P-value \\
\hline Tumor location & & 0.942 & & & & 0.998 & & \\
\hline Tumor size (cm) & $1.48(1.18-1.85)$ & $<0.001$ & $1.48(1.18-1.86)$ & $<0.001$ & $1.47(1.21-1.80)$ & $<0.001$ & & \\
\hline $\begin{array}{l}\text { Tumor size } \\
(\geq 2.4 \mathrm{~cm} \text { vs. }<2.4 \mathrm{~cm})\end{array}$ & & 0.995 & & & $6.12(1.35-27.76)$ & 0.019 & $5.53(1.17-26.30)$ & 0.031 \\
\hline Histologic type & & 0.276 & & & & 0.968 & & \\
\hline Lauren type & & 0.902 & & & & 0.582 & & \\
\hline Method of operation & & 0.519 & & & & 0.085 & & \\
\hline $\begin{array}{l}\text { Gastrectomy } \\
\text { (total vs. subtotal) }\end{array}$ & $13.33(2.99-59.25)$ & 0.001 & $6.78(1.31-35.24)$ & 0.023 & $21.46(5.85-78.67)$ & $<0.001$ & $19.28(4.96-74.90)$ & $<0.001$ \\
\hline
\end{tabular}

$\mathrm{OR}$, odds ratio; $\mathrm{Cl}$, confidence interval.

The mean tumor size was $2.7 \mathrm{~cm}$ in patients with negative frozen biopsy results, and $5.5 \mathrm{~cm}$ in patients with positive frozen biopsy results $(\mathrm{P}=0.035)$. Although it was not statistically significant, the proportion of undifferentiated histologic type was higher in patients with positive frozen biopsy results. Tumor size of patients who had to undergo re-resection was significantly larger than that of those who did not have the re-resection procedure $(4.9 \mathrm{~cm}$ vs. $2.6 \mathrm{~cm}, \mathrm{P}=0.032$ ).

The ROC and AUC of tumor size in predicting the possibility of re-resection were 0.715 (95\% confidence interval [CI], 0.58-0.84; 
$\mathrm{P}=0.007)$. The cutoff value of the tumor size was $2.4 \mathrm{~cm}$ with a Youden index of 0.362 . The multivariate analysis in Table 3 shows that tumor size was significantly associated with positive frozen biopsy results (odds ratio [OR], 1.48; 95\% CI, 1.18-1.85; $\mathrm{P}<0.001$ ), and tumor larger than $2.4 \mathrm{~cm}$ was significantly associated with re-resection (OR, 5.53; 95\% CI, 1.17-26.30; $\mathrm{P}=0.031$ ).

\section{DISCUSSION}

We compared the results of frozen biopsy, additional resection rate, and PRM to investigate whether intraoperative AXR was useful in determining adequate resection lines. This study showed no significant difference in outcome between patients who underwent intraoperative AXR and those who did not. This finding suggests that intraoperative AXR is not necessary to obtain safe resection margins during laparoscopic or robotic gastrectomy and intracorporeal anastomosis.

In total laparoscopic or robotic surgery with endoscopic clipping, it is difficult to confirm the location of the clip by touching the serosal surface in the operation field. To complement this, endoscopic dye injection, intraoperative endoscopy, laparoscopic ultrasonography, and intraoperative radiography have been proposed.

Indigo carmine dye injection through endoscopy can cause a spreading out of the lesion over time after injection and can lead to blurring of the border. The indocyanine green (ICG) method requires additional expensive equipment for ICG detection and cannot be used in patients with contrast allergies [9]. In addition, uncommon complications may occur after endoscopic staining, such as abscess forming peritonitis, fat necrosis, inflammatory gastritis, and chemical peritonitis $[7,10,11]$. Laparoscopic ultrasonography and intraoperative endoscopy require specific endoscopic skills and devices. In addition, because of the inability to obtain a visual field during surgery due to bowel distension, additional procedures are needed to decompress the gas in the intestine or to clamp the proximal jejunum so that air does not descend into the jejunum [6].

Despite the disadvantage of additional endoscopy once before surgery, preoperative endoscopic clipping is often used to localize the non-palpable EGC lesion, to obtain an adequate resection line, and minimize the unnecessary length of the PRM [4]. To determine the location of the preoperative applied clip during the operation, we frequently use intraoperative AXR. This method does not require specific skills or devices compared to other methods and is a cost-saving method [8]. However, there are concerns of unnecessary radiation exposure to the patient and the medical team. In addition, by omitting this process, we can reduce the op- erating time for the radiographer. As a result, we investigated the necessity of intraoperative AXR.

In addition to identifying clips during the operation and avoiding tumor positive frozen biopsy results and re-resection procedures, it is important for endoscopists to clip the anatomical "proximal" margin and not the "lateral or around" margin of the tumor. To easily identify the location of the clips, the standard view of EGD is important. These standard EGD views should include the esophagogastric junction, angle, or gastric rugae. Although it was not statistically significant, undifferentiated gastric cancer was more frequent in patients with positive frozen biopsy. Undifferentiated tumors may have poor demarcated margins, and this factor can make it difficult for endoscopists to identify the appropriate place to clip. Furthermore, in previous studies, large tumors were significantly associated with positive resection margin, and large size was a risk factor for this result $[12,13]$. We also found that large tumor was significantly associated with re-resection. Based on these results, we suggest that endoscopic clipping should be performed $1 \mathrm{~cm}$ or more proximal to the tumor.

Since we did not have detailed protocol for endoscopic clipping in our center, important standard views of EGD with clips could not be retrieved, which may affect the results after gastrectomy. In addition, it is unfortunate that we did not have the data of accurate measurements between the predicted location of the endoscopic clip and anatomical landmarks of the stomach during laparoscopic or robotic surgery.

We analyzed a small number of cases performed by only two surgeons (JHL and JYA) in this study. Therefore, there might be selection and information bias with a limitation in generalization of this result to other hospitals or surgeons.

In addition, there are differences in anastomosis methods between groups and the position of tumor is higher in group B, which may act as a bias in the analysis. In group A, 212 of 251 patients (84.5\%) were JHL's patients, and in group B, all 80 patients (100\%) were JYA's. According to the operator's preference for anastomosis method, group A had a high Billroth II anastomosis rate $(75.7 \%)$ and group B had a high Billroth I anastomosis rate (65\%). In comparison with Billroth I, Billroth II anastomosis would often not require intraoperative AXR, because it is possible to obtain enough PRM even if gastrectomy is performed without intraoperative AXR. And in tumor location, although there was no statistically significant difference between the two groups, group B (93.8\%) had a higher tumor location than group A (84.1\%). If the location of the tumor was not high enough to clearly determine the resection line with the preoperative AXR alone, no additional intraoperative AXR would be necessary.

In conclusion, intraoperative AXR is an unnecessary method 
for obtaining safe PRM with negative frozen biopsy results, only when it is possible to predetermine the resection line with preoperative AXR. Also, we suggest that the endoscopic clip should be placed $1 \mathrm{~cm}$ or more proximally, especially in large, poorly differentiated cancers. The location of the clips should be estimated by correlation with preoperative AXR and standard views of the EGD.

\section{CONFLICT OF INTEREST}

No potential conflict of interest relevant to this article was reported.

\section{ACKNOWLEDGMENTS}

JHL contributed to the conception and critical revision of this study. JES and SEO collected and analyzed the data and drafted the work. JYA, MGC, TSS, JMB, and SK ensured that questions related to the accuracy or integrity of any part of the work were appropriately investigated and resolved. All authors gave final approval of the version to be published.

\section{REFERENCES}

1. Japanese Gastric Cancer Association. Japanese gastric cancer treatment guidelines 2014 (ver. 4). Gastric Cancer 2017;20:1-19.

2. Kim HH, Han SU, Kim MC, Kim W, Lee HJ, Ryu SW, et al. Effect of laparoscopic distal gastrectomy vs open distal gastrectomy on longterm survival among patients with stage I gastric cancer: the KLASS-01 randomized clinical trial. JAMA Oncol 2019;5:506-13.

3. Japanese Gastric Cancer Association. Japanese classification of gastric carcinoma: 3rd English edition. Gastric Cancer 2011;14:10112.

4. Ryu KW, Lee JH, Choi IJ, Bae JM. Preoperative endoscopic clipping: localizing technique of early gastric cancer. J Surg Oncol 2003;82: 75-7.

5. Hur H, Son SY, Cho YK, Han SU. Intraoperative gastroscopy for tumor localization in laparoscopic surgery for gastric adenocarcinoma. J Vis Exp 2016;(114):53170.

6. Hyung WJ, Lim JS, Cheong JH, Kim J, Choi SH, Song SY, et al. Intraoperative tumor localization using laparoscopic ultrasonography in laparoscopic-assisted gastrectomy. Surg Endosc 2005;19:1353-7.

7. Hurlstone DP, Fujii T. Practical uses of chromoendoscopy and magnification at colonoscopy. Gastrointest Endosc Clin N Am 2005; 15:687-702.

8. Kim HI, Hyung WJ, Lee CR, Lim JS, An JY, Cheong JH, et al. Intraoperative portable abdominal radiograph for tumor localization: a simple and accurate method for laparoscopic gastrectomy. Surg Endosc 2011;25:958-63.

9. Ushimaru Y, Omori T, Fujiwara Y, Yanagimoto Y, Sugimura K, Yamamoto $\mathrm{K}$, et al. The feasibility and safety of preoperative fluorescence marking with indocyanine green (ICG) in laparoscopic gastrectomy for gastric cancer. J Gastrointest Surg 2019;23:468-76.

10. Coman E, Brandt LJ, Brenner S, Frank M, Sablay B, Bennett B. Fat necrosis and inflammatory pseudotumor due to endoscopic tattooing of the colon with india ink. Gastrointest Endosc 1991; 37: 65-8.

11. Park SI, Genta RS, Romeo DP, Weesner RE. Colonic abscess and focal peritonitis secondary to india ink tattooing of the colon. Gastrointest Endosc 1991;37:68-71.

12. Sun Z, Li DM, Wang ZN, Huang BJ, Xu Y, Li K, et al. Prognostic significance of microscopic positive margins for gastric cancer patients with potentially curative resection. Ann Surg Oncol 2009;16: 3028-37.

13. Wang SY, Yeh CN, Lee HL, Liu YY, Chao TC, Hwang TL, et al. Clinical impact of positive surgical margin status on gastric cancer patients undergoing gastrectomy. Ann Surg Oncol 2009;16:2738-43. 